\title{
INVESTIGACIONES
}

\author{
ANGELA CAMARGO URIBE \\ CHRISTIAN HEDERICH MARTÍNEZ*
}

\section{ANÁLISIS DE DOS TAREAS DE ACCESO AL LÉXICO ${ }^{1}$}

\author{
Las palabras son mágicas. \\ Cuando se presentan \\ uno no puede hacer otra cosa \\ que leerlas \\ (Prinzmetal y cols.,1986)
}

\section{Resumen}

El artículo reporta los resultados de una experiencia en la cual se intenta aportar evidencia frente al problema de si existe una ruta fonológica indirecta o una ruta visual directa para el acceso al léxico durante la lectura de palabras. Si bien la evidencia indica la existencia de una ruta fonológica, los resultados pueden interpretarse de manera alternativa, proponiendo procesos más complejos para la tarea de acceder al sentido de una palabra.

\section{Abstract}

This paper reports the results of an experience that tried to obtain evidence to solve the problem of whether there is an indirect phonological route or a direct visual route for the lexical access to written words. While the evidence indicates the existence of a phonological route, the results can be interpreted in a different way, postulating more complex processes when the task requires access to the meaning o a word.

\section{Palabras claves:}

Procesamiento verbal, léxico, rutas de acceso léxico, rimas, clases semánticas.

En culturas escritas, la lectura es una actividad que se aprende desde muy temprano en la vida, lo que permite la construcción de rutinas de procesamiento cognitivo automáticas, fijas y muy rápidas para el reconocimiento de las palabras escritas. Es este proceso el que, en estrecha interacción con aspectos formales

\footnotetext{
* Profesores Universidad Pedagógica Nacional

${ }^{1}$ El presente artículo retoma algunos resultados del proyecto de investigación "Estilo cognitivo y lectura de palabras", realizado por los autores como requisito para ascenso en el escala fón. Sin embargo, ese proyecto de investigación no tiene como tema principal el que este trabajo pretende des arrollar.
} 
(sintácticos), semánticos y pragmáticos del discurso, permite la comprensión de textos.

En el lenguaje de la psicología del procesamiento de la información, la actividad de reconocer palabras corresponde al proceso de acceso al léxico. El acceso al léxico tiene lugar en el momento en que todas las propiedades de una palabra (fonológicas, morfológicas, sintácticas y semánticas) se encuentran disponibles para su tratamiento por el sistema cognitivo (Alvarez, Alameda y Domínguez, 1999). Para lograr esto ${ }^{2}$, el sistema necesita, de un estímulo perceptivo inicial a partir del cual el conocimiento que se posee sobre la palabra se recupere de la memoria de largo plazo (MLP) y se active en la memoria de trabajo (MCP). Es decir, se requiere de un código de acceso.

En el caso específico del reconocimiento de palabras escritas, podría pensarse que este código de acceso estaría conformado por algunas características ortográficas de la palabra. El uso de códigos de acceso de tipo fonológico sería aquí innecesario. Ciertamente, no habría justificación inmediata para la necesidad de utilizar propiedades fonológicas de las palabras para acceder a ellas durante la lectura, si se tiene en cuenta que ésta es una actividad cuya esencia es la construcción de sentido a partir de información perceptual visual.

Curiosamente, los modelos psicolingüísticos que se han construido para describir el proceso de acceso al léxico durante la lectura, más que dividirse entre aquellos que consideran el acceso fonológico innecesario y aquellos que lo consideran posible, se dividen entre aquellos que lo consideran absolutamente necesario y aquellos que lo consideran optativo.

En efecto, un buen número de modelos psicolingüísticos de reconocimiento de palabras proponen la hipótesis de que, a fin de acceder a una palabra escrita, es absolutamente necesario acceder primero a su representación fonológica, (Coltheart, 1978; McCusker, Hillinger y Bias, 1981). De acuerdo con esta hipótesis, la recodificación fonológica de la información visual (la ortografía de la palabra), mediante la aplicación de reglas de conversión grafema-fonema, es un paso previo obligatorio para acceder al resto de propiedades de la palabra, incluido su significado.

Los investigadores en favor de esta propuesta plantean que es necesario cons truir modelos de acceso léxico generales, es decir, que puedan explicar el reconocimiento de palabras tanto habladas como escritas. Con este propósito, intentan encontrar un código de acceso común tanto para la lectura como para la audición de palabras. Según estos investigadores, dicho código sería la representación fonológica de las palabras. Apoyando esta línea de ideas, se encuentra el argumento de que el reconocimiento auditivo de palabras es un proceso bastante más universal que el reconocimiento visual (existen lenguas sin

\footnotetext{
${ }^{2}$ Hablamos aquí del caso en que la lectura de una palabra se realiza aisladamente, es decir cuando la palabra no se encuentra inserta en un enunciado que hace parte de un texto.
} 
sistema de escritura) y que, siendo la lectura una habilidad que se aprende con posterioridad al desarrollo del lenguaje hablado, el procesamiento de información escrita sería, así mismo, un proceso que depende del procesamiento auditivo de las palabras (Belinchón y otros, 1992).

Por su parte, otros modelos de acceso al léxico proponen una hipótesis alternativa. Autores como Forster (1979), entre otros, han propues to que el acceso léxico durante la lectura de palabras sigue dos rutas posibles: una ruta directa de reconocimiento de ras gos visuales y letras, y otra ruta indirecta, en donde, una vez reconocido el código ortográfico, és te se recodifica fonológicamente para que las siguientes operaciones de reconocimiento puedan darse. A la primera ruta se le llama ruta visual y a la segunda ruta fonológica. (Rubenstein, 1971, Meyer, Schvaneveldt y Ruddy, 1974).

De acuerdo con esta hipótesis llamada "de ruta dual", la vía fonológica sería una alternativa posible, pero no obligatoria, para la identificación de palabras escritas. La elección de esta ruta estaría deteminada por factores relativos a la naturaleza de la información por procesar. Es común encontrar, por ejemplo, que las palabras con ortografía irregular tarden más en ser reconocidas que las palabras con ortografía regular. Este hecho se explica postulando que, en el caso de las palabras ortográficamente irregulares, se utilizaría la ruta fonológica con la aplicación de la operación extra de conversión grafémico-fonémica. (Perfetti y Hogaboan, 1975; Baron y Strawson,1976), mientras que en el caso de palabras con ortografía regular la ruta visual directa sería suficiente. Además de la irregularidad ortográfica, la selección opcional de una u otra ruta se ha asociado con el grado de familiaridad que se tenga con la palabra que se está leyendo. Se ha postulado que las palabras de mayor frecuencia, o más familiares para el lector, tienden a reconocerse por la ruta visual y las de menor frecuencia, o desconocidas, por la ruta fonológica (Seidenberg et al.,1984).

De acuerdo con la evidencia anotada arriba, la ruta visual directa sería la usada "por defecto" por el sistema y la ruta fonológica indirecta sería utilizada en los casos en los que el sistema encuentra dificultades para la ubicación de la secuencia gráfica en el diccionario mental.

Si bien la hipótesis de la ruta única o de recodificación fonológica parece bastante potente atendiendo a su generalidad, que incluso podría apoyarse en argumentos de tipo biológico (el carácter innato del aprendizaje de los sistemas lingüísticos orales), la hipótesis de la ruta dual resulta considerablemente razonable, en tanto explicaría evidencia experimental importante.

Desde nuestro punto de vista, y de acuerdo a lo anteriormente expuesto, la discusión acerca de las rutas que permiten el acceso al significado de las palabras estaría reducida entonces a la postulación de una ruta visual directa para explicar el acceso al léxico de ciertas palabras. Dependiendo de si se logra encontrar o no evidencia sobre la exis tencia de esta ruta, la balanza se inclinaría a favor de una u otra hipótesis. 
Con miras a aportar evidencia en este sentido, el presente artículo reporta los resultados de la aplicación de dos tareas de decisión léxica en las que se intenta aislar dos procesos posibles durante el acceso al léxico: el acceso a la representación fonológica de la palabra y el acceso a la repres entación semántica de la misma.

Un grupo de 25 estudiantes, de ambos sexos, estudiantes de grado 8o. del Instituto Pedagógico Nacional de la ciudad de Bogotá, Colombia, con edades comprendidas entre los 13 y los 15 años constituyó nues tra muestra de sujetos de investigación.

Estos estudiantes llevaron dos tareas de acceso al léxico que pasaremos a explicar a continuación.

\section{Las tareas de acceso al léxico}

Regulamente, un proceso cognitivo como el reconocimiento de palabras se observa experimentalmente mediante la medición de la eficacia operativa del mismo, la cual posee como indicadores básicos la velocidad y la precisión en la respuesta a una tarea de reconocimiento dada (De Vega y Cuetos, 1999). En el contexto de las investigaciones psicolingüísticas esta observación se lleva a cabo mediante el diseño de tareas de decisión léxica (TDL). Una tarea de decisión léxica consiste en presentar al sujeto un estímulo y éste tiene que decidir algo sobre la secuencia presentada (si es una palabra o no, si es una palabra de cierta forma o no, etc.). Para ello debe presionar palancas o botones de "si" o "no". Se suele registrar el tiempo de reacción y los errores cometidos. El primer dato permite deducir la velocidad y el segundo la precisión en la respuesta (Álvarez, Alameda y Domínguez, 1999) ${ }^{3}$.

Las tareas diseñadas para la experiencia que aquí reportamos están basadas en la prueba de emparejamiento físico y nominal de letras o palabras, diseñada por Posner (Posner et al., 1969, citado por Mayer, 1985) y utilizada, entre otros autores, por Kleinman (1975) para identificar rutas privilegiadas en el acceso léxico. Kleinman (1975) realizó un experimento en donde los sujetos tenían que resolver dos tipos de tareas: En la primera, tenían que decidir si dos palabras estímulo tenían significados parecidos, y en la segunda, tenían que decidir si dos palabras rimaban. Mientras realizaban estas dos tareas, los sujetos iban repitiendo, en voz alta, una secuencia de números que escuchaban por

\footnotetext{
${ }^{3}$ Por lo regular, los estímulos se presentan en una pantalla de computador, por lo cual la interfase corresponde a un teclado o a una palanca de "joystick". El programa donde ha sido diseñada la tarea permite el registro automático de respuestas y tiempo de reacción.
} 
auriculares. En el experimento se registraban niveles de corrección de las respuestas de los sujetos ${ }^{4}$.

El procedimiento general consiste en presentar al sujeto parejas de palabras en una pantalla de computador y pedirle que presione el botón "si" o el "no" de acuerdo con ciertas instrucciones iniciales. En la primera tarea (la de rimas), el sujeto debe decidir si las dos palabras riman (por ejemplo: rodillo-tomillo); en la segunda (la de clases semánticas), el sujeto debe decidir si las palabras aluden a elementos de la misma clase (por ejemplo: caballo-conejo).

Dado que el proceso que se intenta observar indirectamente, es decir, el proceso de acceso al léxico, es una tarea bastante sensible a factores de diversa índole tales como la longitud de la palabra, la familiaridad del sujeto con la misma, etc., la selección de los ítems intentó controlar la mayor cantidad posible de los factores identificados como intervinientes en el proceso. Se intentó, en consecuencia, homogeneizar la muestra de palabras de acuerdo con: su longitud (no menos de tres y no más de cuatro sílabas), la familiaridad de las mismas (palabras de uso cotidiano para los sujetos de la muestra) y nivel de concreción de los significados (se evitaron palabras referidas a entidades abstractas).

De estas manera, se eligieron 40 parejas de palabras: las primeras 20, examinaban la tarea de rimas, las segundas 20 , la tarea de clases semánticas. Dentro de cada uno de estos grupos los 20 ítems se distribuyeron de acuerdo con: si las parejas de palabras rimaban o no rimaban y si pertenecían a la misma clase o no. Así, dentro de cada tarea pueden distinguirse: 5 parejas de palabras que riman y pertenecen a la misma clase (respuesta afimativa en las dos tareas); 5 parejas que riman pero no pertenecen a la misma clase (respuesta afimativa en rimas y negativa en clases); 5 parejas que no riman y sí pertenecen a la misma clase (respuesta negativa en rimas y afirmativa en clases) y 5 parejas que no riman y no pertenecen a la misma clase (res pues ta negativa en los dos casos).

\footnotetext{
${ }^{4}$ Los sujetos se equivocaban con más frecuencia en los juicios sobre rimas que en los juicios sobre parecido
} semántico. 
Tabla 1: Ítems de las tareas

\begin{tabular}{|l|l|l|l|l|l|}
\hline \multicolumn{4}{|l|}{ TAREA DE RIMAS } & \multicolumn{3}{l|}{ TAREADE CLASES } \\
\hline Cod & P1 & P2 & Cod & P1 & P2 \\
\hline R01 & tapete & alfombra & C01 & tomate & alicate \\
\hline R02 & bolsillo & amarillo & C02 & remolach & arracach \\
& & & & a \\
\hline R03 & mercurio & anturio & C03 & triángulo & círculo \\
\hline R04 & giras ol & armario & C04 & planeta & cometa \\
\hline R05 & bolero & balada & C05 & pantalón & domilón \\
\hline R06 & patineta & bicicleta & C06 & panaderí & droguería \\
& & & & a & \\
\hline R07 & es fero & ventana & C07 & manzana & durazno \\
\hline R08 & chaqueta & camiseta & C08 & diamante & elefante \\
\hline R09 & carretera & carrilera & C09 & antena & espejo \\
\hline R10 & cerveza & cabeza & C10 & cultivo & huerta \\
\hline R11 & pimentón & conductor & C11 & teclado & manguer \\
& & & & & a \\
\hline R12 & zorrillo & conejo & C12 & bus eta & martillo \\
\hline R13 & llavero & corazón & C13 & sombrilla & montaña \\
\hline R14 & coliseo & estadio & C14 & madera & nevera \\
\hline R15 & pradera & ladera & C15 & tortuga & oruga \\
\hline R16 & galleta & magnolia & C16 & palmada & patada \\
\hline R17 & tornillo & pocillo & C17 & tijeras & repollo \\
\hline R18 & florero & sombrero & C18 & mojarra & róbalo \\
\hline R19 & licuadora & lavadora & C19 & tornado & sentado \\
\hline R20 & pantufla & zapato & C20 & cuchillo & tenedor \\
\hline
\end{tabular}

Las parejas de palabras en el orden en que fueron presentadas para cada tarea se muestran en la tabla 1. Cada ítem se presenta con un código, consistente en una letra y un número de dos dígitos: La letra recuerda la tarea solicitada (R:rimas, C:clases), y el número hace referencia al orden de presentación (desde 01 has ta 20). En adelante nos referiremos a este código para identificar los ítems específicos.

\footnotetext{
${ }^{5}$ Colombianismo de bolígrafo

${ }^{6}$ Colombianismo para referi rse a cierto tipo de autobús. 


\section{Análisis cognitivo de las tareas}

\section{Tarea de rimas}

Desde una perspectiva lingüística, la rima es la repetición o coincidencia sonora que se da al final entre dos o más secuencias verbales, a partir de la última vocal acentuada. Cuando la igualdad sonora engloba a todos los sonidos - tanto vocálicos como consonánticos - se dice que la rima es consonante. En cambio, cuando la igualdad sonora se circunscribe a los sonidos vocálicos se habla de rima asonante. Esta distinción básica entre los tipos de rima fomaliza niveles de similitud fonética entre dos expresiones. La rima consonántica produce efectos sonoros más coincidentes que la rima as onántica.

En el caso de esta tarea concreta, la instrucción dada a cada sujeto no especificaba el tipo de rima que se debía usar para la comparación, por lo cual se supone que el sujeto asume como parámetro de decisión la rima consonántica, cuyo sentido es el más conocido y manejado en contextos no especializados. Por supuesto, no se espera que el sujeto sea capaz de explicar técnicamente el hecho; pero, en la medida en que el fenómeno de la rima es naturalmente aprendido y su noción se utiliza cotidianamente, sí se espera que no haya problemas en la comprensión de la naturaleza de la tarea.

Una secuencia objetiva de acciones cognitivas para la resolución de la tarea de rimas podría ser la que sigue:

- El sistema registra en la memoria de corto plazo (MCP) la secuencia de letras que componen la primera palabra estímulo (ítem léxico de la izquierda de la pantalla).

- Se aplican las reglas de conversión grafema-fonema, que convierten cada letra en el fonema que corresponde de acuerdo con el sistema ortográfico del español. Este proceso tiene como resultado el acceso a la forma fonológica de la primera palabra.

- El sistema regis tra en la MCP la secuencia de letras que componen la segunda palabra estímulo (ítem léxico de la derecha de la pantalla).

- Se aplican las reglas de conversión grafema-fonema, que convierten cada letra en el fonema correspondiente de acuerdo con el sistema ortográfico del español. Este proceso produce como resultado la forma fonológica de la segunda palabra.

- El sistema lleva a cabo la comparación entre las dos formas fonológicas que tiene a su disposición y estima el nivel de similitud fonológica entre ellas, respecto de un umbral específico que para el caso de la tarea de rima. En este proceso, es probable que sectores diferentes de las formas fonológicas de las palabras posean diversos "pesos" relativos al criterio de comparación. Es de suponer, por ejemplo, que la similitud entre las últimas partes de las palabras (después del acento) pos ean mayor relevancia para la decisión en cuestión. El 
resultado permite que el sistema tome una decisión afimativa o negativa frente a la pregunta de si las palabras riman o no.

- Finalmente, la decisión permite ejecutar la rutina de respuesta que ha sido automatizada previamente. Es decir, presionar el botón del "si" o del "no" de acuerdo con el proceso anterior.

Es importante anotar aquí que este no es el único algoritmo de procesamiento posible para la resolución de la tarea cognitiva. Podría considerarse por ejemplo que el sistema accediera y asimilara en la MCP las formas visuales de las palabras, para después proceder a decodificarlas fonológicamente. Este proceso es perfectamente factible a las capacidades del sistema cognitivo humano, y podría ser considerablemente más veloz.

\section{Tarea de clases semánticas}

Desde un punto de vista estrictamente lingüístico, la tarea de decisión de clases semánticas alude al establecimiento de relaciones de sentido siempre presentes en la estructura léxica de una lengua. El sentido de una expresión es, simplemente, el conjunto de relaciones semánticas (de sentido) que se mantienen entre dicha expresión y otra cualquiera. Entre las muchas relaciones de sentido que dos ítems léxicos pueden guardar entre sí, está la de pertenencia a la misma clase o campo semántico. Si asumimos una definición clásica des de la lógica, dos palabras pertenecen a la misma clase si el sentido de ambas "entraña" una tercera que denota la clase natural a la que pertenecen las dos. La relación de entrañamiento, originalmente desarrollada para la lógica de predicados, se utiliza aquí en su aplicación más general para especificar una relación de inclusión del sentido de una palabra dentro de otra. Así por ejemplo, si el sentido de "gato" entraña "animal" y el sentido de "perro" también lo hace, es posible afimar que las palabras "perro" y "gato" pertenecen a la misma clase semántica.

Básicamente, lo que la tarea está pidiendo es que el sujeto identifique para las dos palabras estímulo una tercera palabra que sea parte del sentido de ambas. Por supuesto, y desde una perspectiva lógica general, siempre es posible encontrar una categoría o clase semántica que abarque dos términos, cualesquiera que estos sean. Acudimos entonces a la noción de clase natural. Para la semántica contemporánea de corte cognitivo, todo rótulo lingüístico (las palabras) se construye a través de categorías cognitivas. Es decir, que las unidades lingüísticas son tipos de categorías cognitivas construidas por los individuos a partir de la experiencia cotidiana, que es la que pemite la construcción de las llamadas clases naturales (categorías cuyos miembros comparten la misma es encia). Así, dos términos pertenecen a la misma clase natural (o clase semántica) en la medida en que la experiencia del sujeto haya construido una categoría supraordinada que abarque a los dos. Las clases naturales presentan efectos prototípicos: hay un miembro de la clase que se constituye en el prototipo o punto de referencia desde el cual los demás miembros de la clase son categorizados. 
Puede hablarse incluso de grados o niveles de prototipicidad (algunos miembros de la clase natural son más prototípicos que otros; por ejemplo "paloma" es más prototípico para la clase natural "aves" que "avestruz" o "pingüino"). Entre más prototípica es una categoría, más rápidamente es reconocida como miembro de la clase natural, es más rápidamente aprendida (por los niños) y es mencionada de primeras cuando se pide que se enumeren miembros de una clase natural.

En vista de todo lo anterior, suponemos que en esta tarea de decisión léxica, los sujetos acudirán tanto a su sis tema léxico, organizado de acuerdo a relaciones de sentido, como a su sis tema cognitivo en el cual el mundo ha sido categorizado por medio de clases naturales. Este conocimiento experiencial, categorizado mediante rótulos lingüísticos, es del que echará mano el sujeto para tomar decisiones frente a la tarea.

El análisis objetivo de la tarea de clases semánticas podría describirse como sigue:

- El sistema registra en la MCP la información grafémica correspondiente a la primera palabra estímulo (ítem léxico de la izquierda).

- Si el código de acceso privilegiado por el sistema es de tipo visual, esta información se toma como base para acceder directamente al significado de la palabra. Si, por el contrario, la ruta de acceso es de tipo fonológico, previo al acceso léxico se aplican reglas de conversión grafema-fonema, y posteriomente accede al significado de la palabra.

- El sistema registra en la MCP la información grafémica correspondiente a la segunda palabra estímulo (ítem léxico de la derecha).

- Como en el caso de la primera palabra, si el código de acceso privilegiado por el sis tema es de tipo visual, esta infomación se toma como base para acceder al significado de la palabra. De nuevo, si la ruta de acceso es de tipo fonológico, previo al acceso léxico se aplican reglas de conversión grafemafonema.

- Los dos significados son comparados con respecto de las categorías cognitivas supraordinadas que los componen. Si alguna de estas categorías es compartida por ambos significados, el sis tema toma una decisión afirmativa. Si ninguna de las categorías supraordinadas son compartidas por los dos significados, la decisión es negativa.

- El sistema ejecuta la rutina de respuesta correspondiente al resultado del proceso anterior.

De forma similar a lo que acontece para la tarea de rimas, es probable que esta secuencia operativa presente variaciones. Puede suponerse, por ejemplo, que el sujeto comience la tarea de identificar clases naturales (categorías supraordinadas) en el momento en que accede a la primera de las dos palabras 
estímulo, y que la lectura de la segunda incluya un juicio respecto de la pertenencia a la clase semántica ya identificada.

Como puede observarse, a pesar de que los dos tareas suponen operaciones de acceso léxico a la MLP y tratamiento de la infomación en la MCP para la comparación y la toma de decisiones, los procesos varían considerablemente de una tarea a otra, tanto en el tipo de operaciones que se llevan a cabo como en el tipo de información que se procesa para cumplir el objetivo ${ }^{7}$. Esto es posible y es lo que deseamos que suceda en las condiciones experimentales diseñadas.

Las tareas de la prueba son intentos por aislar experimentalmente y llevar a la conciencia procesos que ocurren simultánea e inconscientemente durante la lectura. No pretendemos haber llegado al esquema ideal de control de variables en el que cada proceso se ejecuta en independencia del otro, lo cual es teórica y prácticamente imposible. Sin embargo, si consideramos haber interpuesto una cierta distancia entre los procesos de acceso fonológico y semántico que nos ayuda al examen y caracterización de cada uno de ellos.

\section{Hipótesis de trabajo}

Recordemos en es te punto que nuestro objetivo es la exploración de la posibilidad de encontrar índices de la existencia de una ruta visual directa para el reconocimiento de palabras. Con esto en mente, el razonamiento que conduciría a la confirmación de la existencia de esta ruta sería el siguiente.

- Si los tiempos de reacción para la tarea de rimas son significativamente similares a los tiempos de reacción de la tarea de clases semánticas, supondremos que los sujetos estarán utilizando una ruta de acceso directo en cada caso, lo cual implicaría la exis tencia de una ruta visual directa usada en el caso en que se necesite, es decir, en la tarea de clases.

- Si, por el contrario, los sujetos se demoran significativamente más en la tarea de clases que en la tarea de rimas, supondremos que la ruta fonológica indirecta fue usada para realizar la tarea de clases, en un momento en que, de acuerdo con la tarea esto no habría sido necesario, es decir, que se estaría encontrando evidencia a favor de la hipótesis de una ruta única de tipo fonológico.

\footnotetext{
${ }^{7}$ Lo que indica esta diferencias es que una actividad aparentemente tan simple como la del tratamiento de palabras de una lengua implica un nivel de complejidad cognitiva considerable
} 


\section{Resultados}

\section{Los tiempos de latencia}

En términos generales, el examen de los tiempos de latencia de respuesta frente a una tarea cognitiva es básico en la deteminación del proceso cognitivo seguido por el sistema en cada tarea. En general, se considera que mayores tiempos de latencia son indicadores de un proceso cognitivo más complejo en el sentido de mayor número de procesos involucrados en el procesamiento.

Un punto tiene especial importancia: en la medida en que no disponemos de un modelo que incorpore el error a la predicción del tiempo de latencia, los casos en los que hubo respuestas erróneas fueron eliminados de los cómputos generales de los tiempos promedios de respuesta.

La gráfica 1 presenta el histograma de frecuencias del tiempo promedio para la resolución de los 40 ítems de la prueba en los 25 sujetos examinados. En términos generales, los sujetos de la prueba se toman en promedio 1.64 segundos para resolver cada ítem. Cabe observar un caso particular, el de Carolina, cuyo tiempo promedio en la resolución de los ítems es considerablemente mayor que el de sus compañeros (2.74 seg.). Desde el punto de vista estadístico este es un caso extremo.

Por su parte, el examen de los tiempos medios por ítem muestra gran variabilidad: los promedios de los tiempos de latencia de respuesta en nuestra muestra, frente a cada uno de los 40 ítems incluidos en el experimento, van desde 1.20 segundos en el caso de los ítems más rápidamente resueltos (ítems R10 y C07), hasta 2.35 seg. en el caso del ítem que demandó mayor tiempo (ítem. C19). Esto significa que el tiempo de procesamiento para la comparación de dos palabras puede duplicarse, dependiendo de diferentes factores. En lo que sigue, examinaremos algunos de los factores que mejor explican esta diferencia de velocidad en la respuesta. 


\section{Gráfica 1: Tiempos por sujeto}

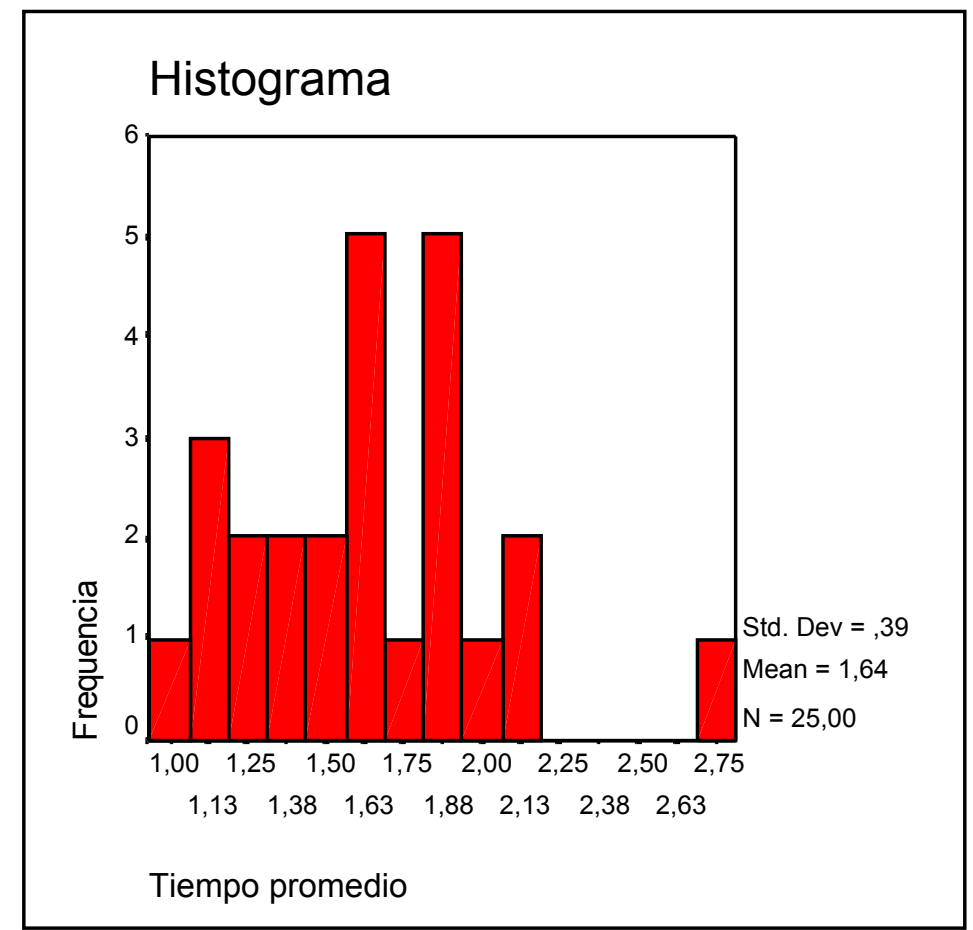

\section{Gráfica 2: Tiempos por ítem}

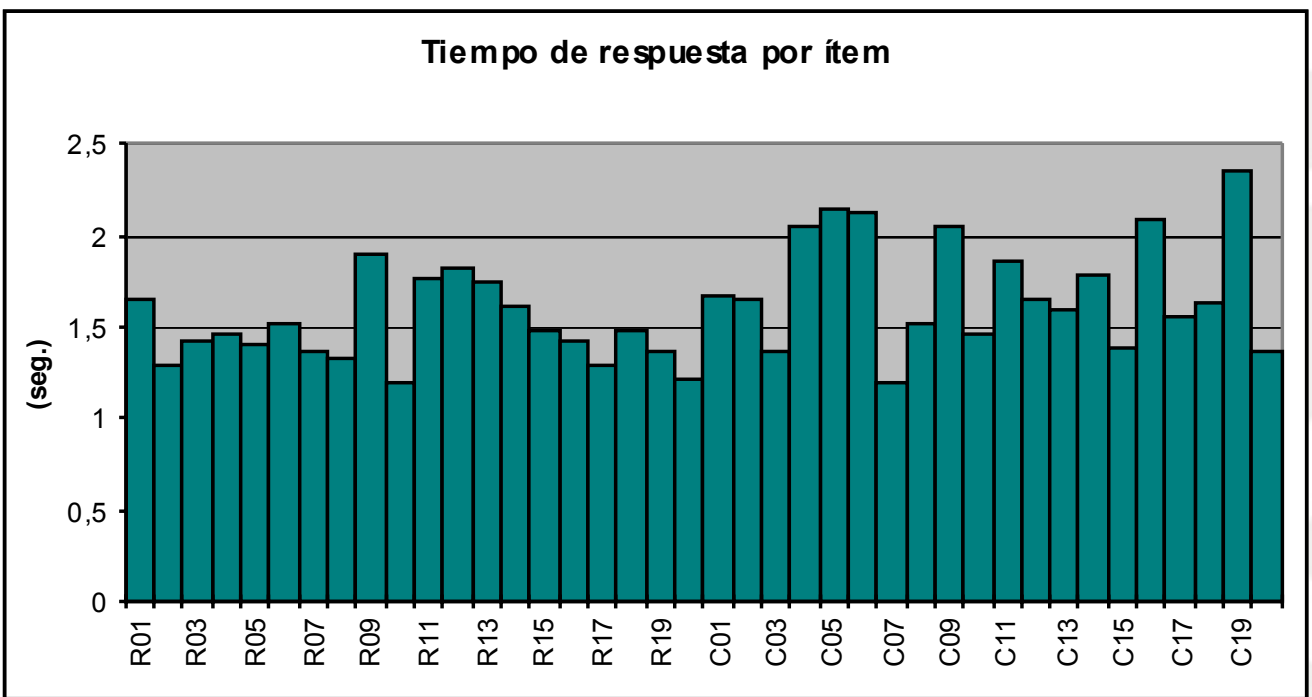

El ítem de mayor tiempo de respuesta fue el C19 (ítem de clases, tornadosentado) que tomó en promedio 2.35 segundos y, en orden decreciente, cinco ítems de similitud de clase C05 (pantalón-domilón, 2.14 seg.), C06 (panadería- 
droguería), C16 (palmada-patada), el ítem C04 (planeta-cometa) y C09 (antenaespejo). En sexto lugar aparece el primer ítem de rimas: R09 (carretera-carrilera).

En el extremo contrario, los ítems de menor tiempo de respuesta promedio son R10 (item de rima: cerveza-cabeza, $1.20 \mathrm{seg}$ ) y C07 (de clase: manzana-durazno, $1.20 \mathrm{seg}$ ). Le siguen cinco items de rima (R20, R02, R17, R08 y R07).

Excepto por el ítem de rimas R09 (carretera-carrilera) los ítems de respuesta más lenta corresponden a la tarea de clases semánticas. En contraste, excepto por el ítem de clases C07 (manzana-durazno), el grupo de ítems de respuesta más rápida corresponden a la tarea de rimas. Estos resultados estarían en consonancia con la hipótesis de una mayor velocidad general de respuesta en la tarea de rimas que en la tarea de clases semánticas y corroboran apreciaciones anteriores sobre el hecho de que el acceso a la forma fonológica de la palabra podría ser más rápido o formar parte del acceso al significado de la misma.

A respecto del ítem atípico de rimas R09 (carretera-carrilera), su mayor lentitud de respuesta podría tener que ver con la longitud de la palabra (véase explicación sobre la influencia de la longitud de la palabra en la velocidad de acceso a la misma) y del ítem atípico C07 (manzana-durazno), su mayor rapidez de res puesta puede tener que ver tanto con la familiaridad de las dos palabras como con la familiaridad de la clases natural que las abarca (frutas).

Como se recordará, de acuerdo con nuestras hipótes is, los ítems de rimas pueden ser más rápidamente procesados (lo que significa que presentarán menores tiempos de latencia de respuesta) que los ítems de similitud de clase.

Los resultados apoyan nuestras hipótesis (véase gráfica 3). En promedio, los ítems que requieren la decisión de similitud semántica toman un mayor tiempo de respuesta (1.7255 segundos), que aquellos que solo requieren de la similitud fonética (1.4885 seg.). La diferencia entre los dos promedios es de 0.2370 segundos, diferencia que resulta estadísticamente significativa en una prueba " $\mathrm{t}$ " $(\mathrm{t}=2.82$, sig. bilateral $=0.008)$.

La diferencia entre los dos tipos de ítems, desde la perspectiva del análisis objetivo de tareas, es que los ítems de similitud de rima no requieren, en sentido estricto, el acceso a la memoria semántica de largo plazo, mientras que los de similitud semántica sí lo requieren. Así, en el supuesto de que, a excepción del acceso semántico, en los dos tipos de ítems se mantengan los mismos procesos, este resultado indicaría que las 2.37 décimas de segundo adicionales serían las requeridas para el acceso semántico. Sin embargo, aunque tal supuesto no puede mantenerse con total certeza, sí debe aceptarse, por el momento, un mayor procesamiento (o más complejo) en los ítems de similitud semántica, directamente relacionado con la necesidad del acceso a la memoria semántica de largo plazo. 


\section{Gráfica 3: Tiempos por tipo de tarea ${ }^{8}$}

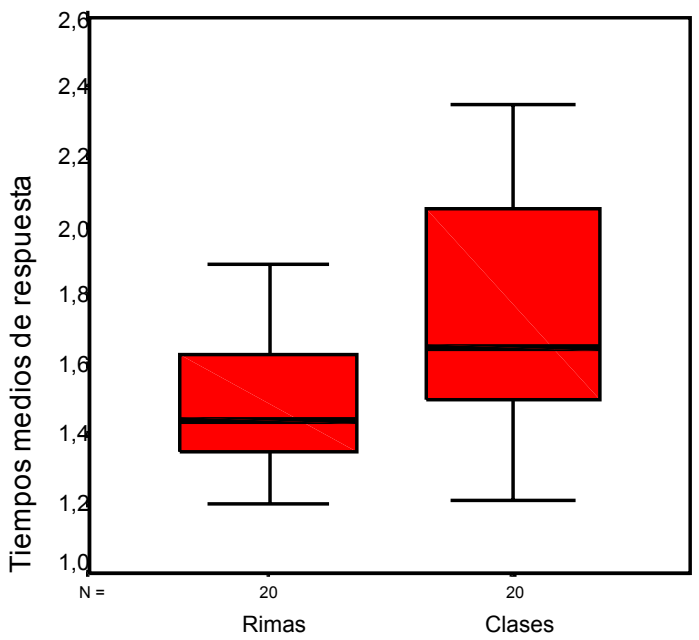

Tipo de tarea

En síntesis, se observa que los tiempos de latencia son mayores en el emparejamiento de clases que en el emparejamiento fonético. Esto podría implicar la diferencia presente en la necesidad de acceso a la MLP semántica presente en las tareas de emparejamiento semántico, pero no necesarias en las tareas de emparejamiento fonético, en donde solo se requiere acceso a la MLP para la descodificación fonética. En este sentido, los resultados apoyan cualquiera de las dos siguientes hipótesis: 1] el acceso fonético a MLP resulta más rápido que el acceso semántico a MLP, y/o que 2] el acceso fonético es apenas una parte del proceso del acceso a la información semántica. Discutiremos esto más adelante.

En cuanto al contraste entre los tiempos promedios de respuesta en ítems de respuesta afirmativa y negativa. En general, es observable una leve diferencia en los tiempos promedios para estos dos grupos de ítems: mientras que los ítems de respuesta negativa toman en promedio $1.68 \mathrm{seg}$., los de respuesta afimativa toman $1.53 \mathrm{seg}$. Esta diferencia es leve y solo alcanza a mostrar significancia en un prueba t unilateral $(t=1.705$, sig. bilateral $=0.096)$.

El examen simultáneo de las dos variables consideradas en la caracterización de los ítems de la prueba sobre el tiempo promedio de latencia de res puesta muestra una fuerte influencia del tipo de tarea (de rimas o de clase) sobre el tiempo promedio y una influencia leve pero observable del tipo de respuesta (verdadero o

\footnotetext{
${ }^{8}$ Los diagramas de cajas muestran información general sobre la mediana, los cuartiles y los valores atípicos y extremos. La caja representa el rango intercuartil que contiene el 50\% de los valores. Las líneas que extienden la caja llegan a los valores más bajos y más altos, excluyendo los casos atípicos (casos entre 1.5 y 3

longitudes de la caja por encima o por debajo) y extremos (casos por encima de 3 longitudes de la caja). La línea gruesa al interior de la caja representa la mediana.
} 
falso) sobre el tiempo. Los datos se presentan en la siguiente gráfica (gráfica 4) y muestran una diferencia de $0.117 \mathrm{seg}$. entre las negaciones y las afimaciones de rimas y de 0.187 entre las de clase. Estas diferencias, dado ya el escaso tamaño de la muestra de ítems no son significativas en ninguno de los dos tipos de ítems (en rimas, $\mathrm{t}=1.33 \mathrm{sig}=0.199$; en clases $\mathrm{t}=1.34 \mathrm{sig}=0.197$ ).

\section{Gráfica 4: Tiempos por tipo de respuesta y tipo de tarea}

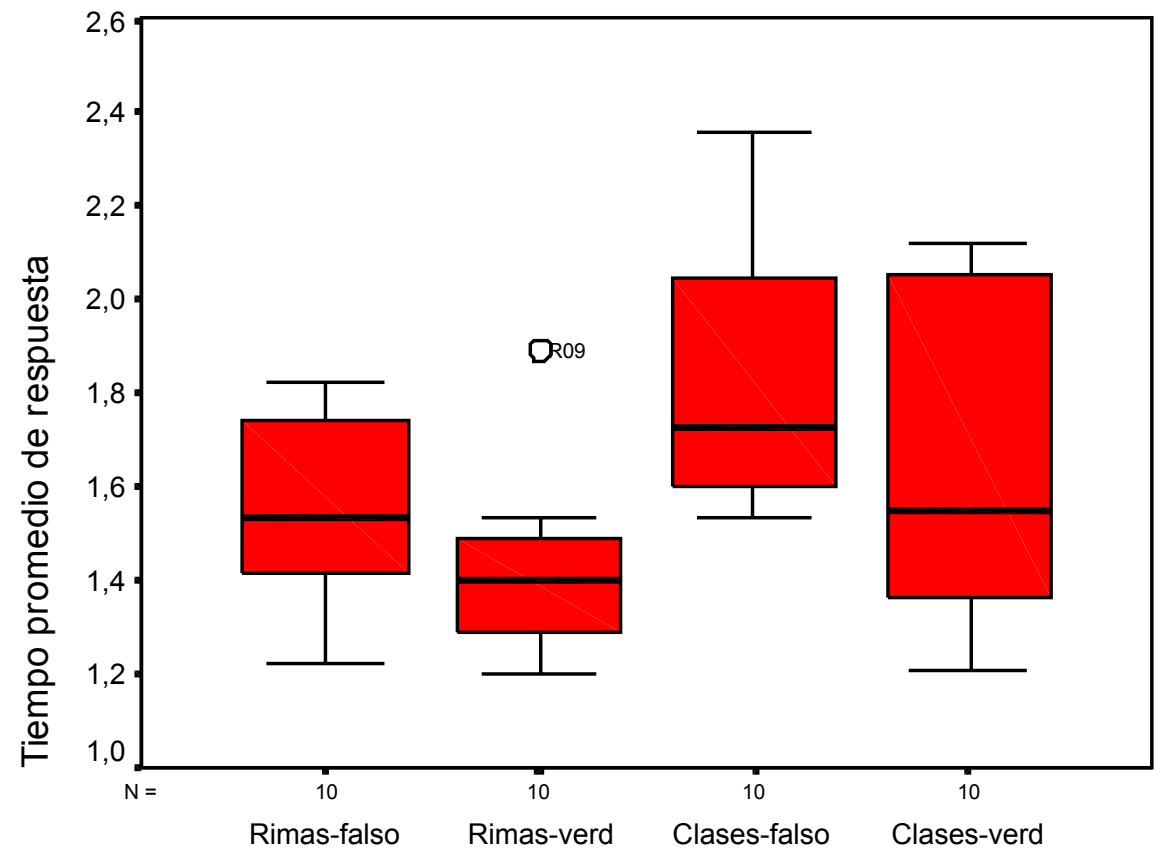

Tipo de ítem

En conclusión, los ítems que requieren respuestas afimativas son procesados de forma ligeramente más rápida que aquellos que requieren respuestas negativas, ya sea que estos sean de rimas o de clases. Los resultados, sin embargo, no son concluyentes por la moderada significancia observada. Si se confimara un resultado en este sentido, esto podría ser explicado desde una óptica chomskiana transformacional (valor afimativo predeterminado en el sistema, y necesidad de proceso adicional en el caso en que se haya concluido el valor negativo (falso) de la proposición.

El examen de la interacción entre la respuesta (afirmativa o negativa) y la clase de la tarea (de similitud fonética o semántica) muestra que, aunque ambos efectos contribuyen en alguna medida a explicar parte importante de la variabilidad (y en particular el que el ítem sea de rimas o de clases), no hay un efecto atribuible a la interacción entre es tos dos factores (véanse los resultados del análisis de varianza en la tabla 2 ). Ello significa que el proceso cognitivo adicional implicado en la negación de una proposición afirmativa opera de forma similar, bien sea que esta proposición se refiera a una similitud fonética o bien sea que esta se refiera a una similitud semántica. Esto es, virtualmente, un indicador de que tal proceso se 
efectúa como parte de una rutina de decisión y ejecución motriz de la respuesta que resulta común a los dos tipos de ítem: la rutina de decisión del valor de verdad de la proposición evaluada, sea esta cual fuere ${ }^{9}$

\section{Tabla 2: Análisis de varianza interacción entre tipo de tarea y tipo de respuesta}

\begin{tabular}{|c|c|c|c|c|c|c|c|}
\hline \multicolumn{8}{|c|}{ ANOVA $^{a, b}$} \\
\hline & & & \multicolumn{5}{|c|}{ UniqueMethod } \\
\hline & & & $\begin{array}{l}\text { Sum of } \\
\text { Squares }\end{array}$ & df & $\begin{array}{l}\text { Mean } \\
\text { Square }\end{array}$ & $\mathrm{F}$ & Sig. \\
\hline \multirow{7}{*}{$\begin{array}{l}\text { Promedios } \\
\text { delatencia }\end{array}$} & \multirow[t]{3}{*}{ Main Effects } & (Combined) & ,793 & 2 & ,396 & 5,836 &, 006 \\
\hline & & fon-da &, 562 & 1 & ,562 & 8,271 &, 007 \\
\hline & & RESPUESTA & ,231 & 1 & ,231 & 3,402 &, 073 \\
\hline & 2-Way Interactions & $\begin{array}{l}\text { fon-cla* } \\
\text { RESPUESTA }\end{array}$ & $1,225 \mathrm{E}-02$ & 1 & $1,225 \mathrm{E}-02$ & , 180 & ,674 \\
\hline & Model & & 805 & 3 & 268 & 3,951 & ,016 \\
\hline & Residual & & 2,445 & 36 & 6,791E-02 & & \\
\hline & Total & & 3,250 & 39 & $8,333 \mathrm{E}-02$ & & \\
\hline
\end{tabular}

a. Promedios de latencia by fon-da, RESPUESTA

b. All effects entered simultaneously

\section{Discusión de resultados}

Hasta el momento, hemos realizado un estudio detallado de los resultados de las tareas en lo que corresponde a lo tiempos de latencia de respuesta observados. Contrastaremos aquí estos resultados con la hipótesis y haremos comentarios adicionales sobre lo encontrado.

La segunda alternativa de la hipótesis que postulamos se vio verificada por los resultados de la prueba. En ella planteábamos que el tiempo requerido para la ejecución de la tarea de rimas iba a ser menor que el tiempo necesario para realizar la tarea de clases semánticas. .En términos procesuales, lo encontrado se traduciría así: el acceso fonético es una parte del proceso del acceso a la información semántica de una palabra; y estaríamos presentando evidencia a favor de una ruta única fonológica para el acceso léxico.

¿Tendríamos que descartar de plano la existencia de una ruta visual directa para el acceso léxico? En realidad no.

\footnotetext{
${ }^{9}$ En términos de la lógica proposicional, hablamos acá de la asignación del valor de verdad de la proposición, o de la aplicación de la "función de verdad" definida en el desarrollo semántico de la lógica. La "semántica", en este contexto formal, no se refiere al uso que hemos venido dándole al término, el cual hace referencia al significado del símbolo. Por esta razón, para evitar con fusiones, evitaremos hablar de semántica de la lógica, restringiendo el uso de lo "semántico" al convencional en la lingüística.
} 
En primer lugar, teniendo en cuenta que la tarea de clases ocurrió después de la tarea de rimas y que en ésta última (la tarea de rimas) era absolutamente necesario realizar un acceso fonológico para lograr el objetivo, es posible que la ruta fonológica haya sido utilizada para la realización de la tarea de clases como consecuencia de una sobremecanización de la misma por parte de los sujetos. En la medida en que venían resolviendo la prueba usando esa ruta, simplemente continuaron utilizándola en el momento en que se cambió de tarea. En este caso estaríamos asistiendo a una especie de habituación del sistema cognitivo en el que, teniendo otras altemativas más eficaces, el sistema maneja la ruta que ya ha utilizado previamente sin detenerse a evaluar cuál sería el camino más expedito para resolver la tarea de clases.

Es posible que esto haya ocurrido sólo en algunos sujetos y que otros sí hubieran cambiado de estrategia. Para verificar esto, tendría que hacerse un anális is de tiempos por sujeto e identificar aquellos cuyos tiempos en la tarea de clases no superaron o fueron menores que en la tarea de rimas. Tendríamos, dado el caso, una situación en que la tendencia a utilizar una sola ruta de acceso sería propia de cierto tipo de sujetos y la tendencia a cariar de tarea de acuerdo con los objetivos sería propia de otro tipo de sujetos ${ }^{10}$.

Relacionado con lo anterior, algunos estudios describen la ruta fonológica, aparentemente menos eficaz para la lectura "de corrido", como estrategia de apoyo en los casos en los que la ruta visual directa fracase por alguna razón (Forster,1990, Kleinman, 1975). En otras palabras, la selección de una u otra ruta de acceso obedecería a factores de tipo estratégico sujetos al control ejecutivo del lector.

En segundo lugar, es posible que, simplemente, estemos frente a dos procesos diferentes, uno para el acceso a información fonológica y otro para el acceso a la información semántica, uno de los cuales, el acceso fonológico, sería más rápido que el otro. En este caso, podríamos simplemente afimar que: el acceso fonético a MLP resulta más rápido que el acceso semántico a la misma. En este caso, se insistiría -tercamente- en la existencia de una ruta visual directa y tendría entonces que entrarse a discutir y explicar por qué la tarea de acceso semántico resulta más lenta, es decir, más compleja, que la tarea de acceso fonológico.

Si tomamos en cuenta los as pectos de la tarea de clases, podría pensarse que para lograrla se requiere de una bús queda exhaus tiva de todas las posibles clases a las que pertenece cada una de las palabras de la pareja a fin de descartar posibles errores en la respuesta. En la tarea de rimas, en contraste, el sujeto solo tendría que juzgar el parecido de las dos palabras que tiene en frente a partir de su reconocimiento de la estructura fonológica de cada una de ellas. La búsqueda en este caso no tendría que ser tan larga para descartar el parecido.

\footnotetext{
${ }^{10}$ Resultados en este sentido se han encontrado tomando como variable el estilo cognitivo de los sujetos. Tales resultados se encuentran consignados en otro documento próximo a publicarse.
} 
Una manera de descartar esta segunda posibilidad de explicación de los resultados sería encontrando una tarea que controlara las múltiples posibilidades de parecido semántico entre dos palabras. Podría pensarse, por ejemplo, en una tarea en la que se incluya una clase es pecífica de objetos y se pregunte al sujeto si las dos palabras que tiene en frente pertenecen o no a esa clase. De esta manera, se reducirían los requerimientos de búsqueda exhaustiva a una clase semántica ya dada y se mantendría intacta la necesidad de acceder al sentido de cada palabra. Por supuesto, este sería un problema para resolver en experiencias futuras.

\section{BIBLIOGRAFÍA}

AL VAREZ, ALAMEDA Y DOMINGUEZ (1999) El reconocimiento de las palabras : procesamiento ortográfico y silábico. En: Manuel de Vega y Fernando Cuetos. Psicolingüística en Español. Madrid : Trotta.

BARON Y STRAWSON (1976) Use of orthographic and word-specific knowledge in reading words aloud. Journal of Experimental Psychology: Human Perception and Performance, 2, 386-393.

BELINCHÓN, M. et al. (1992) Psicología del Lenguaje. Teoría e Investigación. Madrid: Trotta.

COLTHEART, M. (1978) Lexical access in simple reading tasks. En: G. Underwood (ed.) Strategies of information processing. Londres: Academic Press.

DE VEGA, Manuel y CUETOS, Fernando (1999) Introducción. Los desafíos de la Psicolingüistica. EN: M. De Vega y F. Cuetos. Psicolingüística del Español. Madrid: Trotta. Pp 43-52.

FORSTER, K. (1979) Accessing the mental lexicon. En: R.J. Wales y E. Walker (Eds.), New Approaches to Language Mechanisms. Amsterdam: North Holland.

FORSTER, K. (1990) Lexical processing. En D.N. Osherson y H. Lasnik (Eds.) Language, Cambridge, MA.: The MIT Press

HUNT, E. (1986) Capacidad Verbal., En: Sternberg, R. (De.) Las Capacidades Humanas. Un enfoque desde el procesamiento de la información. Barcelona: Labor. 
KLEINMAN (1975) Speech recoding in reading. Journal of Verbal Learning and Verbal Behavior, 14, 323-339.

MAYER, R. (1985) El futuro de la psicología cognitiva. Madrid: Alianza.

McCUSKER, L.X., HILLINGER, M.L. y BIAS, L.G. (1981) Phonological recoding and reading, Psychological Bulletin, 89, 217-245.

MEYER, SCHVANE VELDT y RUDDY (1974) Function of graphemic and phonemic codes in visual word recognition, Memory and Cognition, 2, 309-321.

PERFETTI y HOGABOAN (1975) The relationship between single word decoding and reading comprehension skill, Journal of Educational Psychology, 67, 461469.

PRINZMENTAL, W., TREIMAN, R y RHO, S.H. (1986) How to see a reading unit. Journal of Memory and Language, 25, 461-475.

RUBENSTEIN $\mathrm{H}$ et al. (1971) Evidence for phomenic recoding in visual recognition. Journal of Verbal Learning and Verbal Behavior, 14, 392-397.

SEIDENBERG et al. (1984) Modularity and lexical access. En Y Gopnik y M. Gopnik (eds.), From Models to Modules. Norwood, N.J.: Ablex. 